\title{
Three-Dimensional Dynamics of Narrow Planetary Rings
}

\author{
Eugene I. Chiang \& Christopher J. Culter \\ Center for Integrative Planetary Sciences \\ Astronomy Department \\ University of California at Berkeley \\ Berkeley, CA 94720, USA \\ echiang@astron.berkeley.edu, cculter@uclink.berkeley.edu
}

\begin{abstract}
Narrow planetary rings are eccentric and inclined. Particles within a given ring must therefore share the same pericenter and node. We solve for the threedimensional geometries and mass distributions that enable the Uranian $\alpha$ and $\beta$ rings, and the Saturnian Maxwell and Colombo (Titan) rings, to maintain simultaneous apsidal and nodal lock. Ring self-gravity, interparticle collisions, and the quadrupole field of the host planet balance each other to achieve this equilibrium. We prove that such an equilibrium is linearly stable. Predictions for the Saturnian ringlets to be tested by the Cassini spacecraft include: (1) ringlet masses are of order a few $\times 10^{19} \mathrm{~g}$, (2) surface mass densities should increase from ring midline to ring edges, and (3) rings are vertically warped such that the fractional variation of inclination across the ring is of order 10\%. Analogous predictions are made for the Uranian rings. Simultaneous apsidal and nodal locking forces the narrowest portion of the ring - its "pinch," where self-gravitational and collisional forces are strongest - to circulate relative to the node, and introduces previously unrecognized time-varying forces perpendicular to the planet's equator plane. We speculate that such periodic stressing might drive kilometer-scale bending waves at a frequency twice that of apsidal precession; such flexing might be observed over a few weeks by Cassini.
\end{abstract}

Subject headings: celestial mechanics — planets and satellites: individual (Uranus, $\alpha$ and $\beta$ rings, Saturn, Maxwell and Colombo rings)

\section{INTRODUCTION}

The $\alpha$ and $\beta$ rings of Uranus are eccentric and inclined (Elliot et al. 1984; French et al. 1991). The mean eccentricity, $\bar{e}$, of each $\operatorname{ring}$ is $(0.761 \pm 0.004) \times 10^{-3}$ and $(0.442 \pm$ 
$0.003) \times 10^{-3}$, respectively. The mean inclination, $\bar{I}$, of each ring with respect to the equator plane of Uranus is $0.0152 \pm 0.0006 \mathrm{deg}$ and $0.0051 \pm 0.0006 \mathrm{deg}$, respectively. To maintain an observed eccentricity and inclination, each ring must be composed of particles that share the same longitude of pericenter and same longitude of ascending node. The alignments of apsides and of nodes must simultaneously follow from a delicate balance of forces due to the gravitational field of the planet, ring self-gravity, and interparticle collisions. Obtaining a complete equilibrium solution for a ring's three-dimensional geometry and mass distribution has been a goal of dynamicists for decades. The solution bears directly on ring ages and origins (see, e.g., Goldreich \& Tremaine 1979a; Chiang 2003).

The Maxwell and Colombo ringlets are Saturnian analogs of the Uranian $\alpha$ and $\beta$ rings (Porco 1990). ${ }^{1}$ The mean eccentricities of the Saturnian ringlets, but not their mean inclinations, are measured from Voyager spacecraft observations. Table 1 summarizes observed ring parameters.

Shepherd satellites are expected to confine narrow rings and maintain the latter's sharp edges (Borderies, Goldreich, \& Tremaine 1982), but no satellite has yet been observed to accompany any of the aforementioned ringlets. This state of affairs promises to change with the arrival of the Cassini spacecraft to Saturn in 2004. Narrow rings and their attendant shepherds furnish the most accessible laboratories we have for studying disk-satellite interactions. Their study informs problems on grander scales, including migration of planets within circumstellar disks (see, e.g., Chiang 2003).

The steady-state condition of apsidal alignment, combined with measurements of the ring eccentricity profile, $e(a)$, where $a$ is the semi-major axis of a ring streamline, can be employed to calculate the ring surface density, $\Sigma(a)$. First attempts at this calculation account for the planetary quadrupole field and ring self-gravity, but omit the effects of interparticle collisions (Goldreich \& Tremaine 1979b, hereafter, the "standard self-gravity model"). Chiang \& Goldreich (2000, hereafter CG00) restore the latter effects to demonstrate how collisions near ring boundaries can dramatically raise the ring masses required for apsidal alignment. Surface densities derived by accounting for pressure forces exceed those derived without pressure forces by 1-2 orders of magnitude; the larger masses can be reconciled with occultation measurements and with classical theories of ring shepherding (CG00). A more careful treatment of pressure forces in the ring interior is undertaken by Mosqueira \& Estrada (2002), who reach the same qualitative conclusions as those of CG00.

All the works cited above consider only apsidal alignment and neglect nodal alignment. Nodal alignment, but not apsidal alignment, is studied by Borderies, Goldreich, \& Tremaine

\footnotetext{
${ }^{1}$ The Colombo ringlet is also known informally as the Titan ringlet.
} 
(1983a), who consider ring self-gravity and planetary gravity, but neglect collisions. Ring eccentricities are set to zero in their analysis.

The ring's true surface density profile must be simultaneously reconciliable with both the alignment of apsides and the alignment of nodes; the horizontal structure of a narrow ring is entwined with its vertical structure. This paper seeks to simultaneously treat apsidal and nodal alignment while accounting for the full panoply of forces due to the planetary quadrupole field, ring self-gravity, and interparticle collisions. In $\S 2$, we derive equilibrium ring surface densities and vertical geometries that lock the apsides and nodes of a given ring. We apply our solutions to the $\alpha$ and $\beta$ rings of Uranus, and the Maxwell and Colombo ringlets of Saturn. In $\S 3$, we present a proof that circular, nodally locked rings are linearly stable to perturbations to their inclinations and nodes. The beginnings of such a proof can be found in Borderies, Goldreich, \& Tremaine (1983b); here, we state the arguments more completely and explicitly. In $\S 4$, we discuss our results, highlighting the future impact of the Cassini spacecraft on studies of narrow rings and unresolved theoretical issues.

\section{EQUILIBRIUM}

Our procedure for deriving the mass and 3-dimensional structure of a narrow ring is summarized as follows. The range of semi-major axes spanned by the ring, the eccentricity profile $[e(a)]$, and the mean inclination $(\bar{I})$ are assumed to be given. From $e(a)$, we compute the surface density profile, $\Sigma(a)$, by enforcing apsidal alignment across the ring and by accounting for planetary oblateness, ring self-gravity, and interparticle collisions. ${ }^{2}$ This com- $^{2}$ putation is described in detail in $\S 2.1$. Next, from $\Sigma(a)$ and $\bar{I}$, we compute the inclination profile, $I(a)$, by enforcing nodal alignment across the ring and by accounting for planetary oblateness and ring self-gravity but not interparticle collisions. The computation of $I(a)$ is described in $\S 2.2$. Finally, in $\S 2.3$, we gauge a posteriori the extent to which our neglect of collisional stresses in the derivation of $I(a)$ is justified.

Our computational procedure assumes the ring is characterized by a time-independent argument of pericenter, $\omega$. This means the narrowest portion of the ring (its pericenter) lies at a fixed angle relative to the ring's node on the equator plane of the planet. Strictly

\footnotetext{
${ }^{2}$ The Colombo ringlet has the added complication that it inhabits a 1:0 apsidal resonance established by the Saturnian satellite, Titan; the ring's apsidal precession frequency matches the mean motion of Titan. It is straightforward to show that the contribution of Titan to the differential apsidal precession rate across the Colombo ringlet is small compared to the differential apsidal precession induced by the quadrupole field of Saturn. We will therefore neglect perturbations by Titan on the Colombo ringlet in this paper.
} 
speaking, the assumption of stationary $\omega$ is invalid, since

$$
\dot{\omega}=\dot{\tilde{\omega}}-\dot{\Omega}=2 \dot{\tilde{\omega}} \neq 0
$$

The common precession rate of the longitude of pericenter of all apsidally aligned streamlines, $\dot{\tilde{\omega}}$, is set by the quadrupole field of the central planet and is positive (in the direction of increasing true anomaly). The common precession rate of the longitude of ascending node of all nodally aligned streamlines, $\dot{\Omega}$, is also set by the planetary quadrupole field, is identical in magnitude to $\dot{\tilde{\omega}}$, and is negative. That $\dot{\omega} \neq 0$ implies that the pericenter of the ring circulates relative to the node on the planet's equator plane; since self-gravitational forces are largest at pericenter, the contribution to differential nodal precession from ring selfgravity cannot be time-independent. Our hope is that this complication does not introduce serious errors into our calculation of the time-averaged ring geometry; we point out below where our assumption of fixed argument of pericenter is employed, explore in a preliminary but quantitative manner the consequences of its violation in the Appendix, and speculate about its true effects in the discussion section $(\S 4)$.

\subsection{Surface Density}

We divide the ring into a set of $2 N$ wires and compute the mass of each wire by imposing the steady-state condition of apsidal alignment. The $2 N$ wires are equally spaced in semimajor axis; $N$ wires lie at semi-major axes smaller than that of the ring midline, and $N$ wires lie at greater semi-major axes. Our calculation differs from that of CG00 only in the treatment of collisional forces. We restrict ourselves here to describing this difference; for background details, see CG00.

CG00 highlight the importance of interparticle collisions at ring boundaries and account crudely for their effects by introducing force terms in the equations of motion for "endwires" located within a collisional mean free path, $\lambda$, of either ring boundary. No account is made of collisional stresses in the ring interior (regions displaced many mean free paths from either boundary) in the quantitative model they present. Here we improve upon their calculation by making such an account, although still in a crude and prescriptive manner. We take the solution of CG00 for $\Sigma(a)$ and derive from it a collisional acceleration everywhere within the ring:

$$
\mathbf{C}(a, f)=-\frac{1}{\Sigma} \frac{\partial\left(\Sigma c^{2}\right)}{\partial a} \frac{S}{1-q_{e} \cos f} \hat{\mathbf{r}}
$$


where $\hat{\mathbf{r}}$ points in the radial direction, $q_{e}=a d e / d a$ is the dimensionless eccentricity gradient, and $f$ measures true anomaly. The factor $1-q_{e} \cos f$ is proportional to the local radial spacing between wires (see, e.g., Goldreich \& Tremaine 1979b). Following CG00, we adopt a constant $q_{e}(a)=\overline{q_{e}}$ across the entire ring. The velocity dispersion, $c$, and a dimensionless factor, $S$, are described as follows.

We prescribe the following profile for the square of the velocity dispersion,

$$
c^{2}(a)=c_{i}^{2}+c_{b}^{2} \exp \left(-\left|a-a_{b}\right| / w_{r}\right)
$$

where $c_{i}, c_{b}$, and $w_{r}>0$ are the same constants as employed by CG00, and $a_{b}$ is the semi-major axis of the ring boundary that is nearest a given wire at $a$. According to (3), the velocity dispersion decreases from either ring boundary towards the ring interior over a lengthscale $w_{r}$. The physics underlying the enhancement of velocity dispersion near ring boundaries is elucidated by Borderies, Goldreich, \& Tremaine (1982) and estimates for $c_{i}$, $c_{b}$, and $w_{r}$ are derived by CG00. The actual values we employ are contained in Table 2 .

The function

$$
S=\exp \left[-2 \lambda /\left(\left|a-a_{b}\right|+\lambda\right)\right]
$$

is a softening parameter that we impose because the true acceleration due to particle collisions near a ring boundary is likely overestimated by the usual hydrodynamic expression for the acceleration due to pressure gradients $\left[-\Sigma^{-1} \nabla\left(\Sigma c^{2}\right)\right]$. Within a few mean free paths, $\sim \lambda$, of the ring edge, the hydrodynamic approximation breaks down and particles behave more ballistically with less regard for large-scale gradients in the surface density. The parameter $S$ quickly grows from $e^{-2}$ to unity as we recede from the ring edge towards the ring midline.

The collisional acceleration, $\mathbf{C}$, is inserted into Gauss's equation for a given ring particle's apsidal precession rate, $d \tilde{\omega} / d t$, and averaged over true anomaly to yield, for a given wire, $\langle d \tilde{\omega} / d t\rangle_{C}$. This collisional contribution to the wire's precession rate adds to other contributions due to planetary oblateness and inter-wire gravity. Expressions for the latter two contributions are supplied by CG00. The condition of apsidal alignment requires that precession rates of all wires be equal to the precession rate of a test particle at the ring midline, and yields $2 N$ linear equations for the $2 N$ wire masses. The solution of this linear system generates a new surface density profile, $\Sigma^{\prime}(a)$. The entire calculation is then repeated: from this new surface density, we calculate a new collisional acceleration profile $\left[\mathbf{C}^{\prime}(a, f)\right]$, a new set of wire precession rates due to pressure forces $\left[\langle d \tilde{\omega} / d t\rangle_{C^{\prime}}\right]$, and a new set of $2 N$ linear equations. In this way, the solution for the surface density is iterated until convergence is 
achieved.

In practice, for model parameters appropriate to the Uranian $\alpha$ and $\beta$ rings and the Saturnian Maxwell and Colombo ringlets (see Table 2), we find that the solution of $2 N=$ 2000-6000 wire masses converges after $\sim 10$ iterations. Because the solution is reflectionsymmetric about the ring midline, only $N=1000-3000$ linearly independent equations need be solved for $N$ distinct wire masses. The derivative in equation (2) is computed numerically using a Savitzky-Golay smoothing filter of order 2 and having a width of 20 wires (Press et al. 1992). The system of $2 N$ linear equations is solved using subroutine DGESV of the LAPACK (Linear Algebra Package) software library. Because many of our parameters such as $c$ and $S$ are only order-of-magnitude estimates, our profiles are probably accurate to factors of a few, at best. Nevertheless, by accounting not only for interparticle collisions near ring boundaries but also for collisions within the ring interior, we may explore the qualitative effects of incorporating the latter and make a first-cut correction to the solutions obtained by CG00.

Computed surface density profiles for the $\alpha, \beta$, Maxwell, and Colombo ringlets are displayed in Figures 1-4, respectively. Near the ring midline, our solutions require less mass than those of CG00, a consequence of local pressure gradients that compress the ring and abet ring self-gravity. By contrast, our computed peaks in surface density near ring boundaries are larger than those of CG00. The reason for this is as follows. As a given peak in surface density is approached from the ring midline, steep and inward-directed pressure forces must be balanced by the outward gravitational attraction of massive endwires on the far side of the peak. These inward-directed forces are neglected by CG00; accounting for them here leads to endwire masses larger than those obtained by CG00.

These same qualitative conclusions are reached by Mosqueira \& Estrada (2002), who employ various prescriptions for collisional stresses that differ from ours. Their surface density profiles deviate from ours by large amounts, sometimes by more than an order of magnitude, reflecting the sensitivity of the shape of the profile to choice of boundary conditions. Some consolation may be had in the finding of Mosqueira \& Estrada (2002) that the total ring mass, $M$, is less sensitive to this choice. The chief shortcoming of all calculations of the surface density profile, including our own, is the prescriptive and slightly arbitrary description of the ring boundary. We have assumed here that $q_{e}$ is constant across the entire ring, even in boundary regions resonantly perturbed by shepherd satellites where streamlines can no longer be described as simple ellipses. See CG00 for a discussion of this point. 


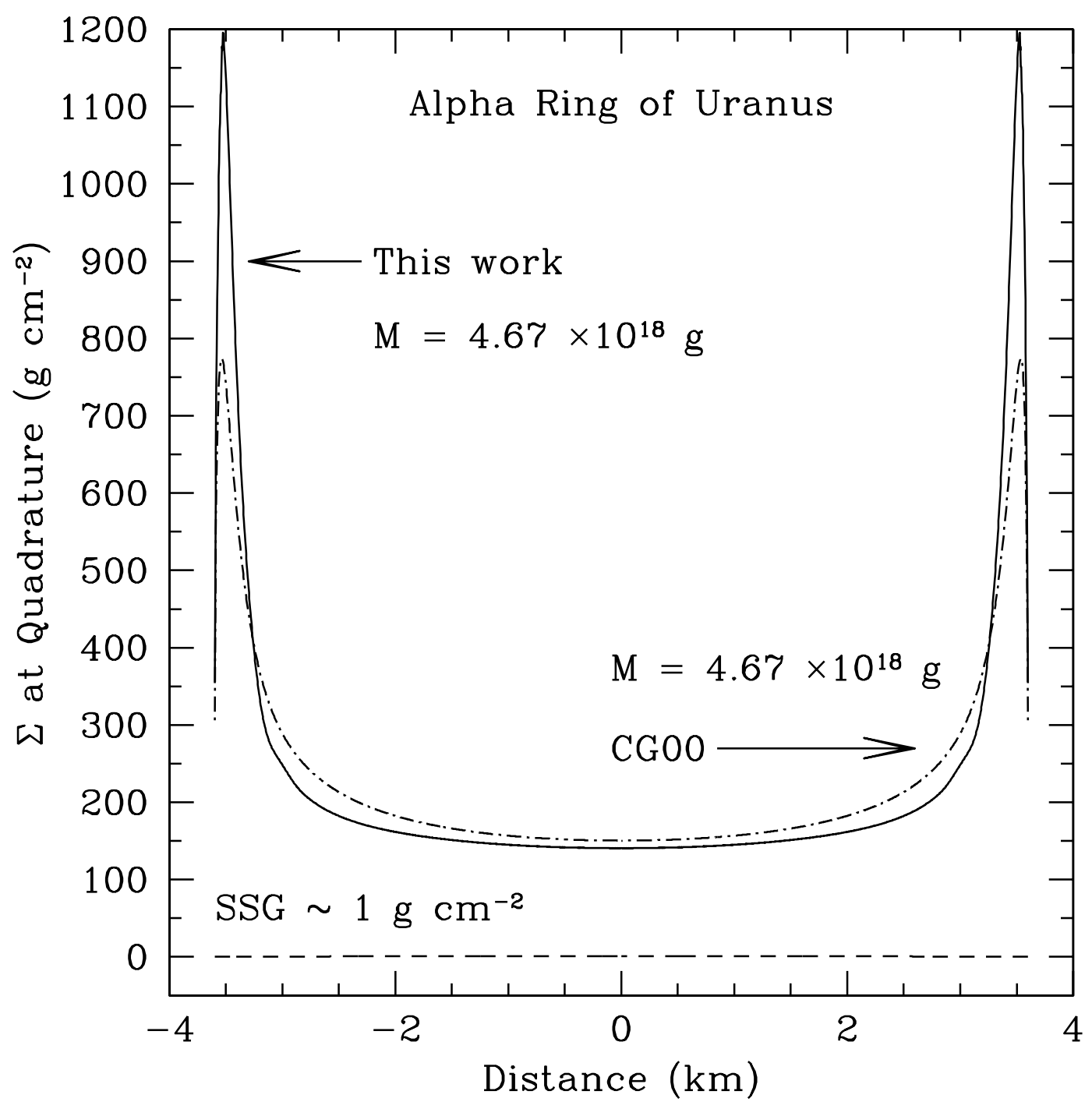

Fig. 1.- Computed surface density profiles for the $\alpha$ ring of Uranus, for $q_{e}(a)=\bar{q}_{e}=0.472$. Including pressure forces in the ring interior lowers the surface density near the ring midline and raises it near the ring boundary, as compared to the model by CG00 who neglect interior pressure gradients. The total mass of the ring, $M$, hardly changes between models, however. The bottom dashed line corresponds to the standard self-gravity (SSG) model of Goldreich \& Tremaine (1979b); it predicts surface densities too low to accord with observations (CG00). 


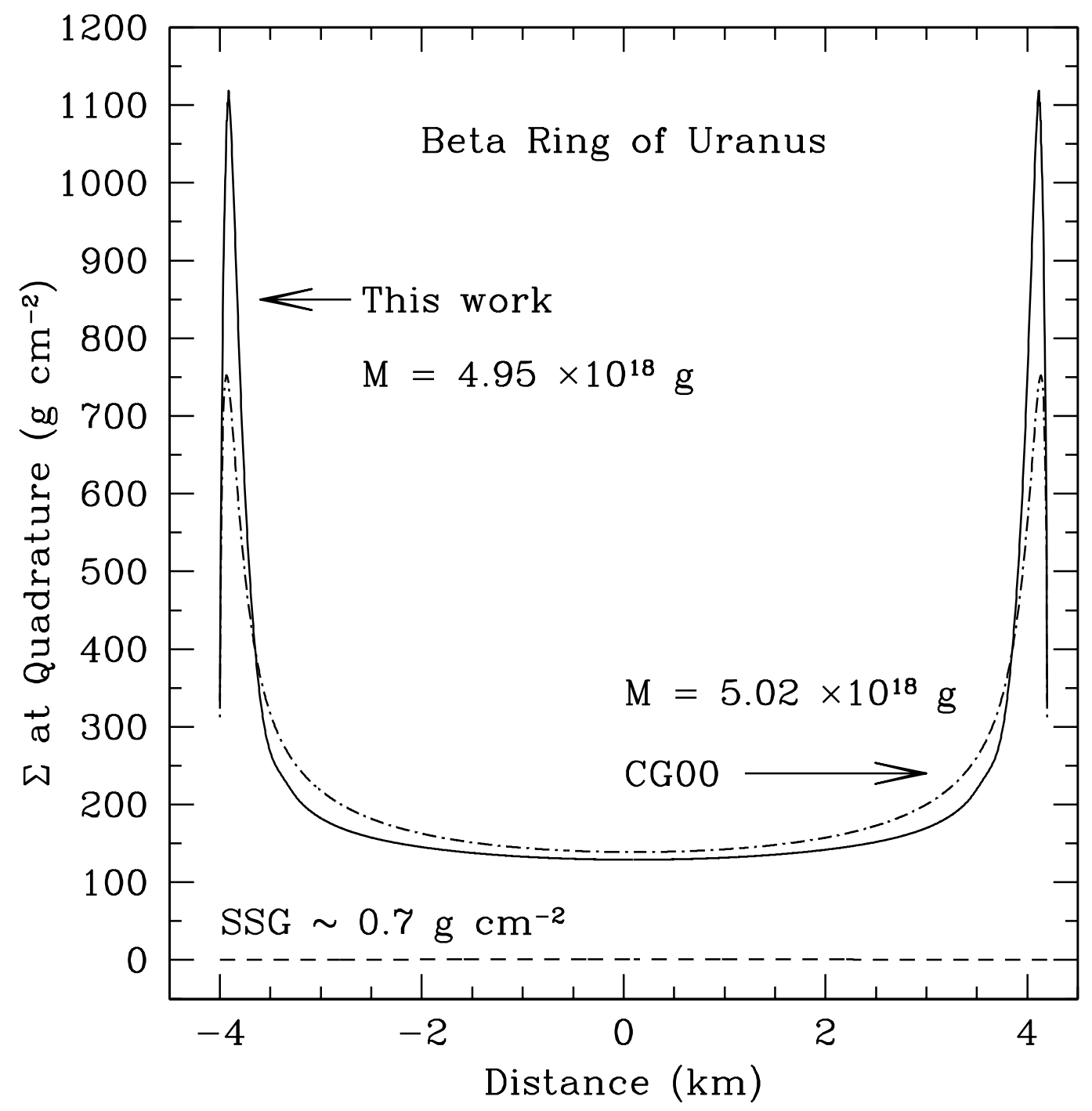

Fig. 2.- Same as Figure 1, but for the $\beta$ ring of Uranus $\left(q_{e}=\bar{q}_{e}=0.370\right)$. 


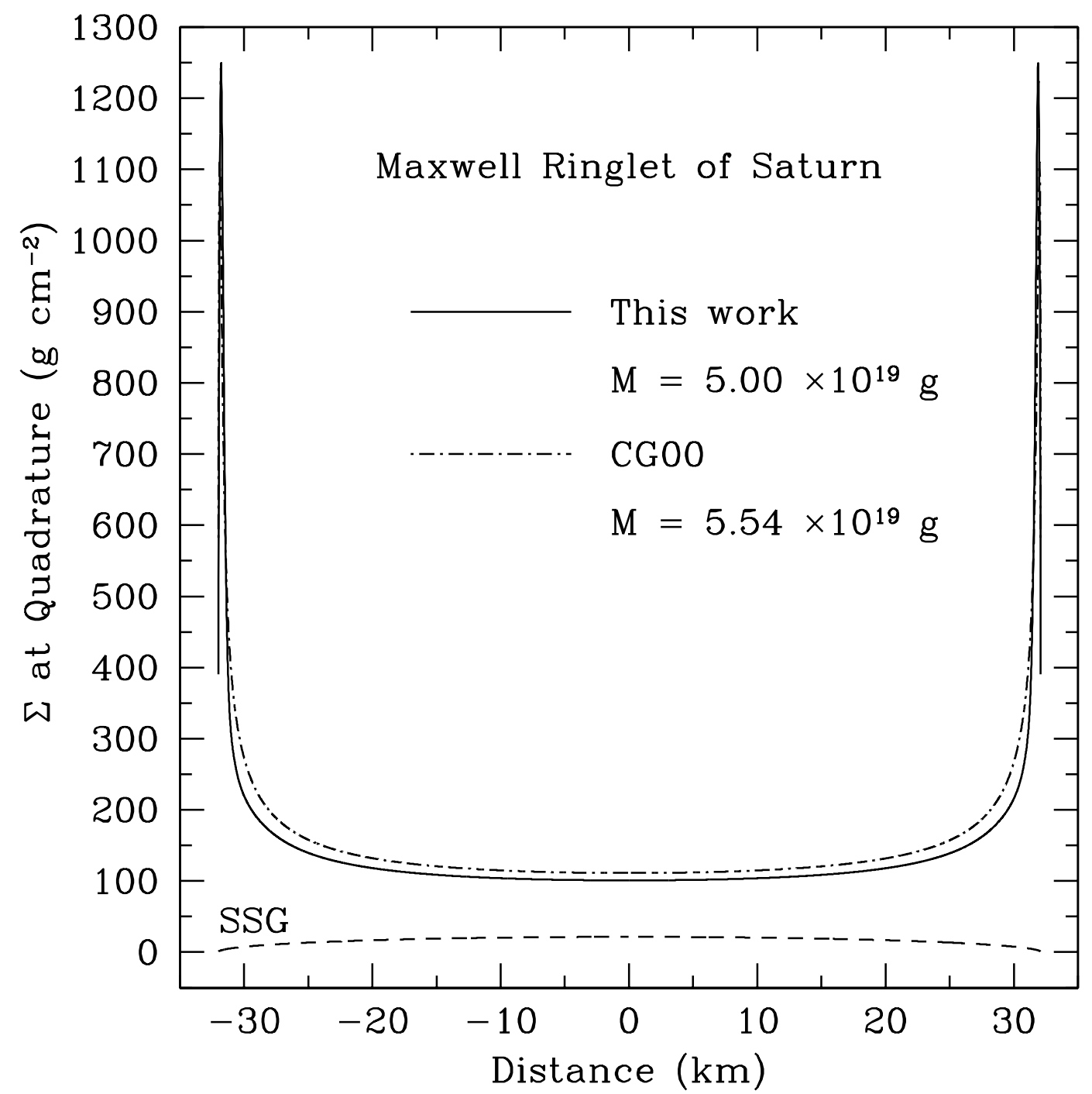

Fig. 3.- Same as Figure 1, but for the Maxwell ringlet of Saturn $\left(q_{e}=\bar{q}_{e}=0.46\right)$. 


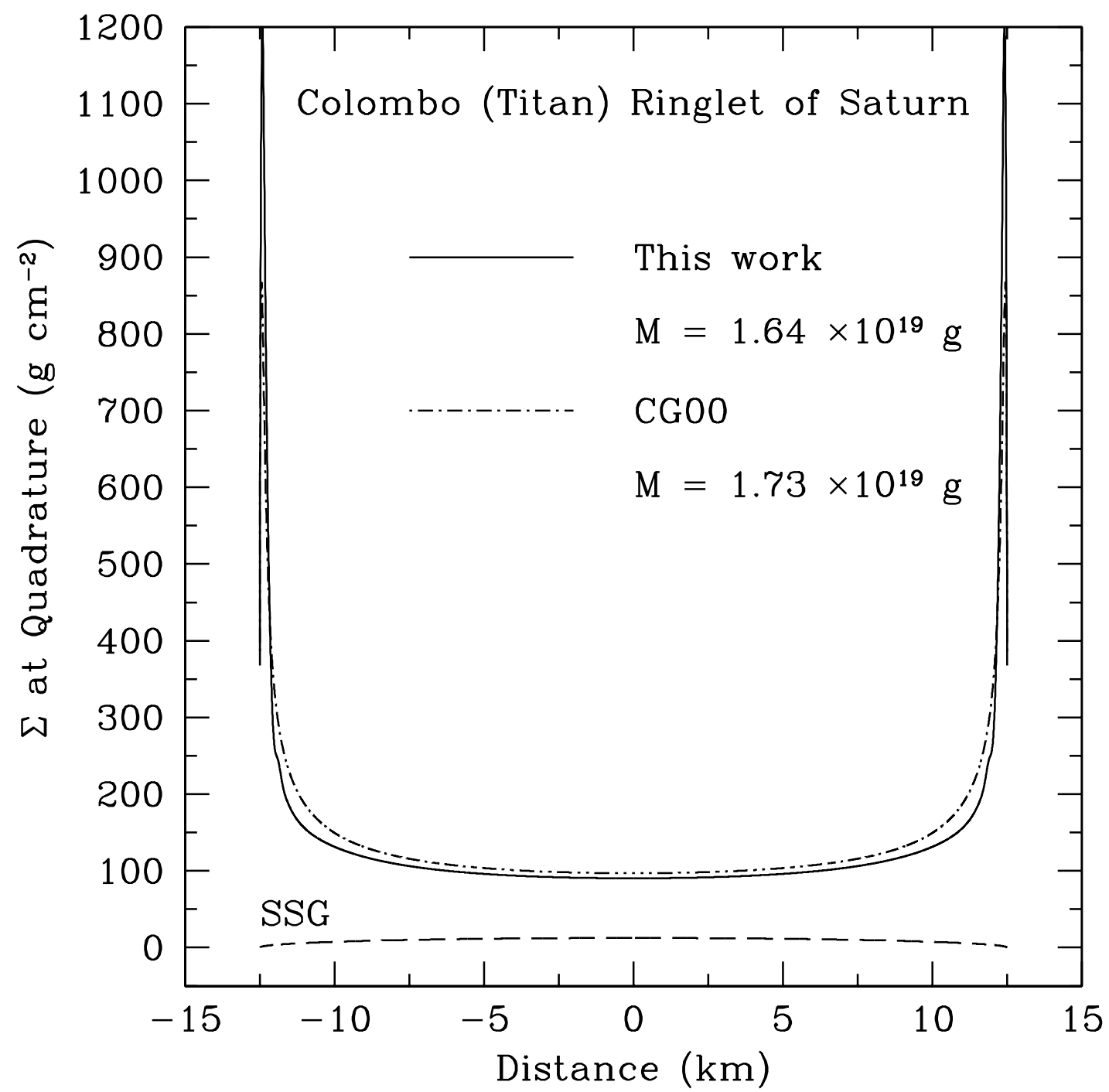

Fig. 4.- Same as Figure 1, but for the Colombo (Titan) ringlet of Saturn $\left(q_{e}=\bar{q}_{e}=0.44\right)$. 


\subsection{Inclination Profile}

Given $\bar{I}$ and $\Sigma(a)$, we compute $I(a)$ by imposing the steady-state condition of nodal alignment. We now discretize the ring into $2 N-1$ wires that share the same longitude of ascending node and solve for the orbital inclination of each wire with respect to the equator plane of the planet. Note that whereas the calculation of the surface density in $\S 2.1$ employs an even number of wires $(2 N)$, here we employ an odd number for the calculation of the inclination profile. The reason for this change of procedure is to have the $N^{\text {th }}$ wire lie on the

ring midline and to assign $I_{N}=\bar{I}$. With this reference point defined, the inclinations of the $N-1$ wires that lie to one side of the midwire may then be computed. Inclinations of the $N-1$ wires that lie on the other side of the midwire follow from reflection anti-symmetry about the midwire.

The nodal precession rate of the $j^{\text {th }}$ wire relative to that of a particle on the ring midline and due to the quadrupole field of the central planet is given by

$$
\Delta_{Q}\langle\dot{\Omega}\rangle_{j}=\frac{21}{4} J_{2} \bar{n}\left(\frac{R_{p}}{\bar{a}}\right)^{2} \frac{a_{j}-\bar{a}}{\bar{a}}
$$

where $\bar{a}$ and $\bar{n}$ are the semi-major axis and mean motion, respectively, of a particle on the ring midline, $J_{2}$ is the usual dimensionless measure of the planetary quadrupole field, $R_{p}$ is the planetary radius, and \langle\rangle denotes a time-average over one orbit. Here $j$ runs from 1 (the wire closest to the planet) to $2 N-1$ (the wire furthest from the planet).

The corresponding differential nodal precession rate due to inter-wire gravity is

$$
\Delta_{G}\langle\dot{\Omega}\rangle_{j}=\frac{\bar{n} \bar{a}}{\pi M_{P} \bar{I}} \sum_{k \neq j}^{2 N-1} \frac{m_{k}}{a_{k}-a_{j}} B q_{k j}
$$

where

$$
q_{k j}=q_{j k}=\bar{a} \frac{I_{k}-I_{j}}{a_{k}-a_{j}},
$$

$M_{P}$ is the mass of the planet, $m_{k}$ is the mass of the $k^{\text {th }}$ wire, and $B$ is a dimensionless function of order unity that depends on the eccentricity profile of the ring, the inclination profile $\left(q_{j k}\right)$, and the arguments of pericenters of the various wires. Following our assumption of a time-independent $\omega$ (see the discussion preceding $\S 2.1$ ), we will take $B=0.77$ constant. 
Though $B$ actually varies with time because $\dot{\omega} \neq 0$, its maximum range of variation is less than a factor of 2 , as we derive in the Appendix.

The $N$ distinct wire masses computed in $\S 2.1$ furnish the set of $\left\{m_{j}\right\}$ used in equation (6) from $j=1$ to $N$; the remaining $N-1$ wire masses are assigned by reflection symmetry across the ring midline.

Steady nodal alignment implies

$$
\Delta_{Q}\langle\dot{\Omega}\rangle_{j}+\Delta_{G}\langle\dot{\Omega}\rangle_{j}=0 \quad\{j=1, \ldots, N-1\}
$$

where we have neglected the contribution from interparticle collisions; the validity of this omission is tested in $\S 2.3$. Embedded in equation (8) are $N-1$ independent equations for the $N-1$ distinct values of $\left\{I_{j}\right\}$.

We proceed to massage equation (8) into a form that permits easy solution. A dimensionless form of the $j^{\text {th }}$ sub-equation reads

$$
\frac{j-N}{2 N-1}+\frac{4 H(2 N-1) M}{21 \pi M_{P} \bar{i} J_{2}}\left(\frac{\bar{a}}{R_{p}}\right)^{2}\left(\frac{\bar{a}}{\Delta a}\right)^{2} \sum_{k \neq j}^{2 N-1} \frac{h_{k}}{k-j} q_{k j}=0
$$

where $\Delta a=a_{2 N-1}-a_{1}$ is the total width of the ring near quadrature, and $h_{k}=m_{k} / M$ is the fraction of the ring's total mass contained in wire $k$. Now each $q_{k j}$ is a linear combination of the $N-1$ independent variables,

$$
x_{i} \equiv q_{i, i+1}=\bar{a} \frac{I_{i}-I_{i+1}}{a_{i}-a_{i+1}} \quad\{i=1, \ldots, N-1\}
$$

as in

$$
q_{j, j+l}=(1 / l) \sum_{i=j}^{j+l-1} x_{i}
$$

Furthermore, for ring self-gravity to balance the planetary quadrupole field, the inclination profile must be reflection anti-symmetric about the ring midline. Then

$$
I_{N+j}-I_{N}=-\left(I_{N-j}-I_{N}\right),
$$

and if $j<N$ and $k>N$, then 


$$
q_{j k}=q_{j N} \frac{j-N}{j-k}+q_{2 N-k, N} \frac{N-k}{j-k} .
$$

It is straightforward to show that by virtue of identities (11)-(13), the summation in equation (9) can be rewritten in terms of the $\left\{x_{i}\right\}$ as

$$
\begin{aligned}
\sum_{k \neq j}^{2 N-1} \frac{h_{k}}{k-j} q_{k j}= & \sum_{i=1}^{i=j-1} x_{i} \sum_{l=1}^{l=i} \frac{h_{l}}{(l-j)|l-j|} \\
& +\sum_{i=j}^{i=N-1} x_{i}\left[\sum_{k=N+1}^{2 N-1} \frac{h_{2 N-k}}{(k-j)^{2}}+\sum_{l=i+1}^{l=N} \frac{h_{l}}{(l-j)|l-j|}\right] \\
& +\sum_{i=1}^{i=N-1} x_{i} \sum_{l=1}^{l=i} \frac{h_{l}}{(2 N-j-l)^{2}} .
\end{aligned}
$$

When equation (14) is substituted into equation (9), we obtain a linear system of $N-1$ independent equations for $N-1$ unknowns, $\left\{x_{j}\right\}$. These are solved numerically using the LAPACK subroutines. The set of inclinations, $\left\{I_{j}\right\}$, are derived from $\left\{x_{j}\right\}$ via (10).

Figures 5 and 6 summarize the results of our calculation for the $\alpha$ and $\beta$ rings of Uranus. These are the only narrow rings whose mean inclinations, $\bar{I}$, have been measured. We predict inclination profiles that are much flatter than those predicted by the standard self-gravity model. The larger ring masses that arise from the inclusion of interparticle collisions enable rings to maintain nodal alignment by warping their geometry by relatively small amounts. The peak-to-peak amplitude of the warp, defined as $a\left(I_{2 N-1}-I_{1}\right)$, is $\sim 7$ meters for the $\alpha$ ring and $\sim 3$ meters for the $\beta$ ring. By contrast, the standard self-gravity model, which employs wire masses derived without regard to interparticle collisions, predicts warp amplitudes of order $1 \mathrm{~km}$.

Figures 7 and 8 describe inclination profiles for the Maxwell and Colombo ringlets of Saturn. The mean inclinations, $\bar{I}$, of these rings with respect to the local Laplacian plane have not been measured. Fortunately, we have found by numerical experiment that the fractional variation of inclination across the ring, $(I-\bar{I}) / \bar{I}$, is independent of the mean inclination. The top panels of Figures 7 and 8 should therefore be fairly accurate. Numerical experiment also reveals that the absolute value of the warp across the ring, $a\left(I_{2 N-1}-I_{1}\right)$, scales linearly with the unknown $\bar{I}$; in computing the absolute value of the warp for the bottom panels, we have simply guessed $\bar{I}=3 \times 10^{-4}$ rad for both rings, values comparable to their observed mean eccentricities. 


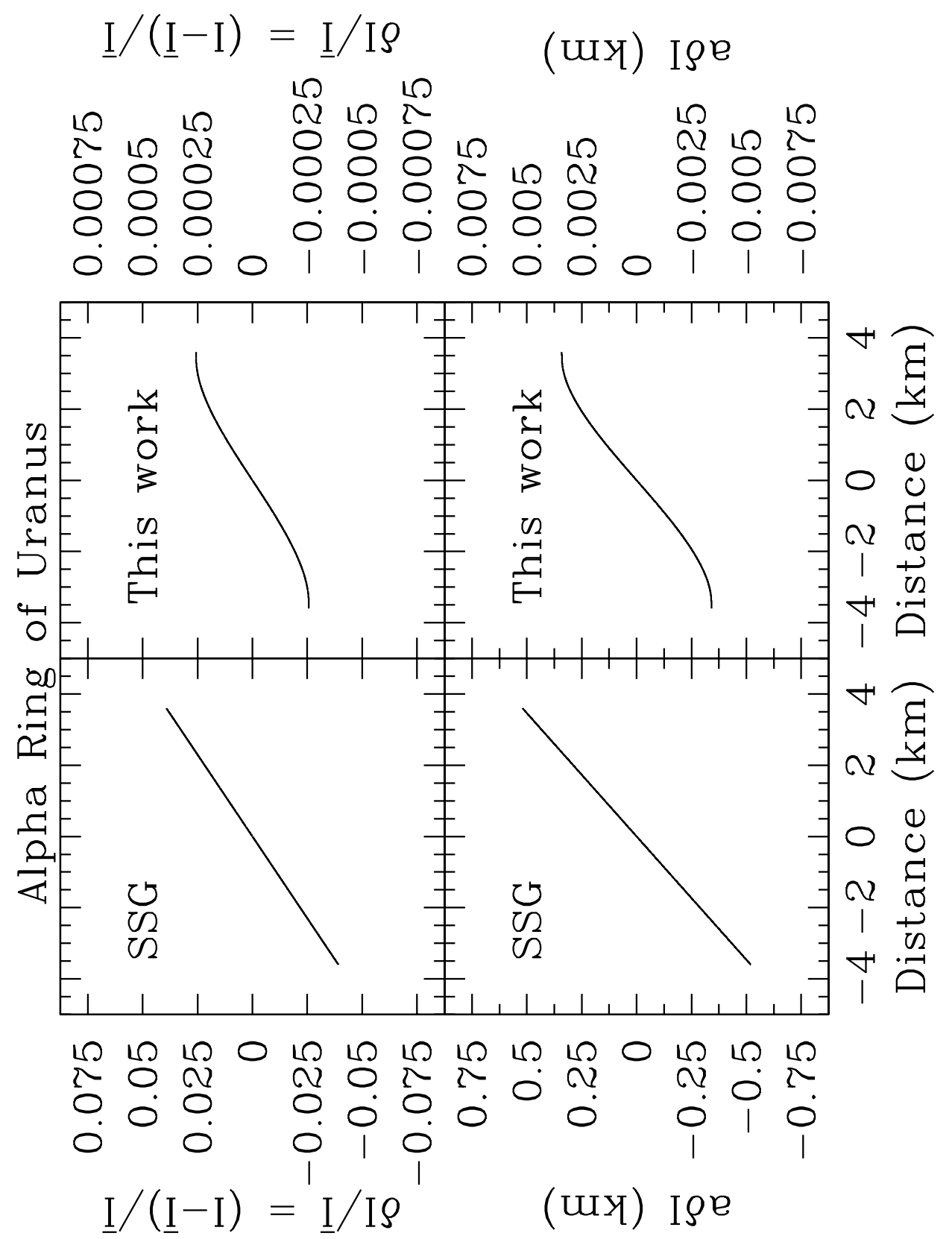

Fig. 5.- Inclination profiles of the Uranian $\alpha$ ring using two different mass models. Righthand panels portray the inclination profile using the surface density profile computed in $\S 2.1$, which accounts for ring self-gravity, the planetary quadrupole field, and interparticle collisions. Left-hand panels correspond to inclination profiles computed under the standard self-gravity model that does not account for interparticle collisions. Upper panels describe the fractional variation in inclination across the ring, while lower panels plot the amplitude of the vertical warp across the ring. The large masses predicted by our work yield much flatter rings than those predicted by the standard self-gravity model; we predict vertical warps of $\sim 7$ meters across a ring that is several kilometers wide. 


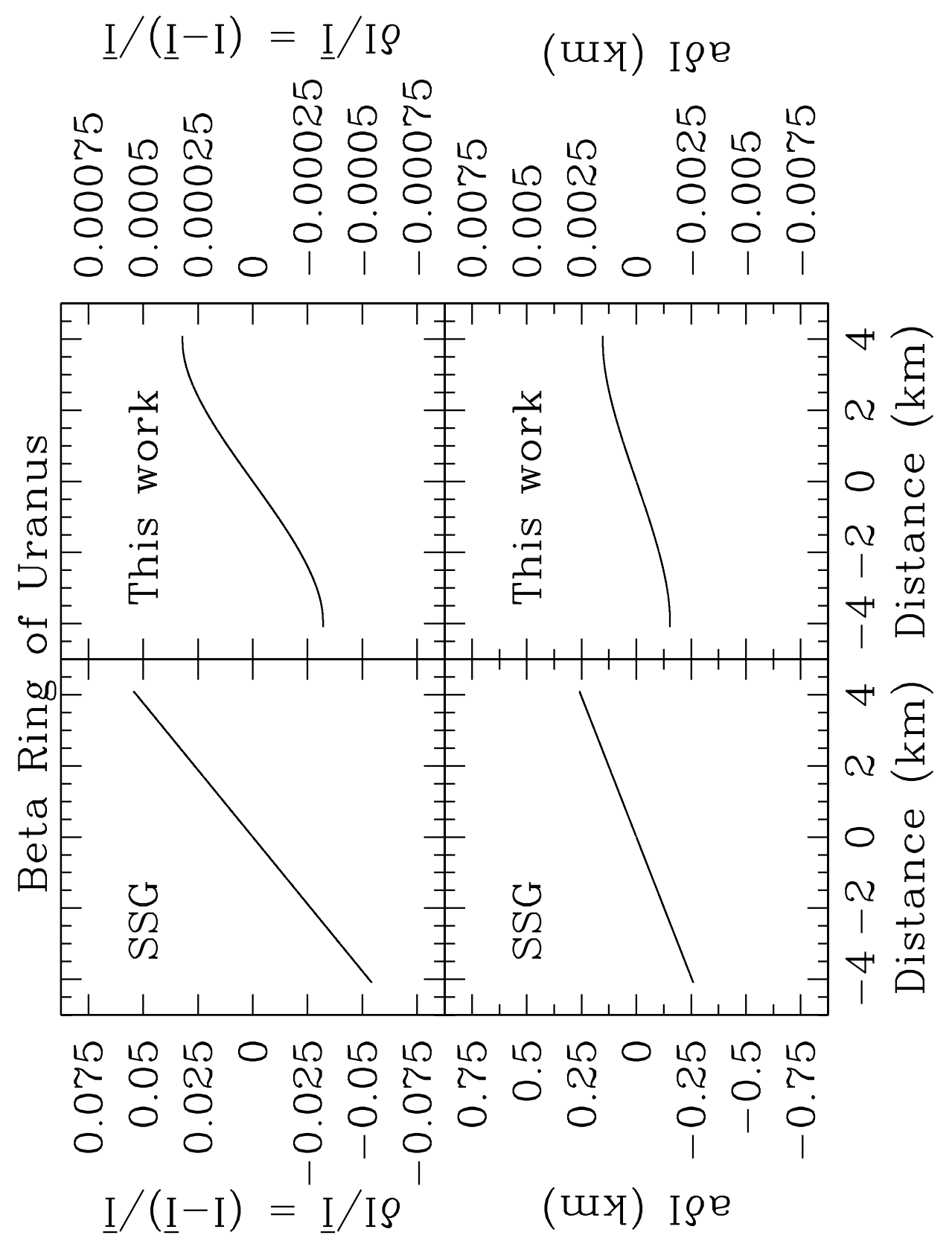

Fig. 6. - Same as Figure 5, but for the $\beta$ ring of Uranus. Warp amplitudes of only $\sim 3$ meters are predicted by our models. 


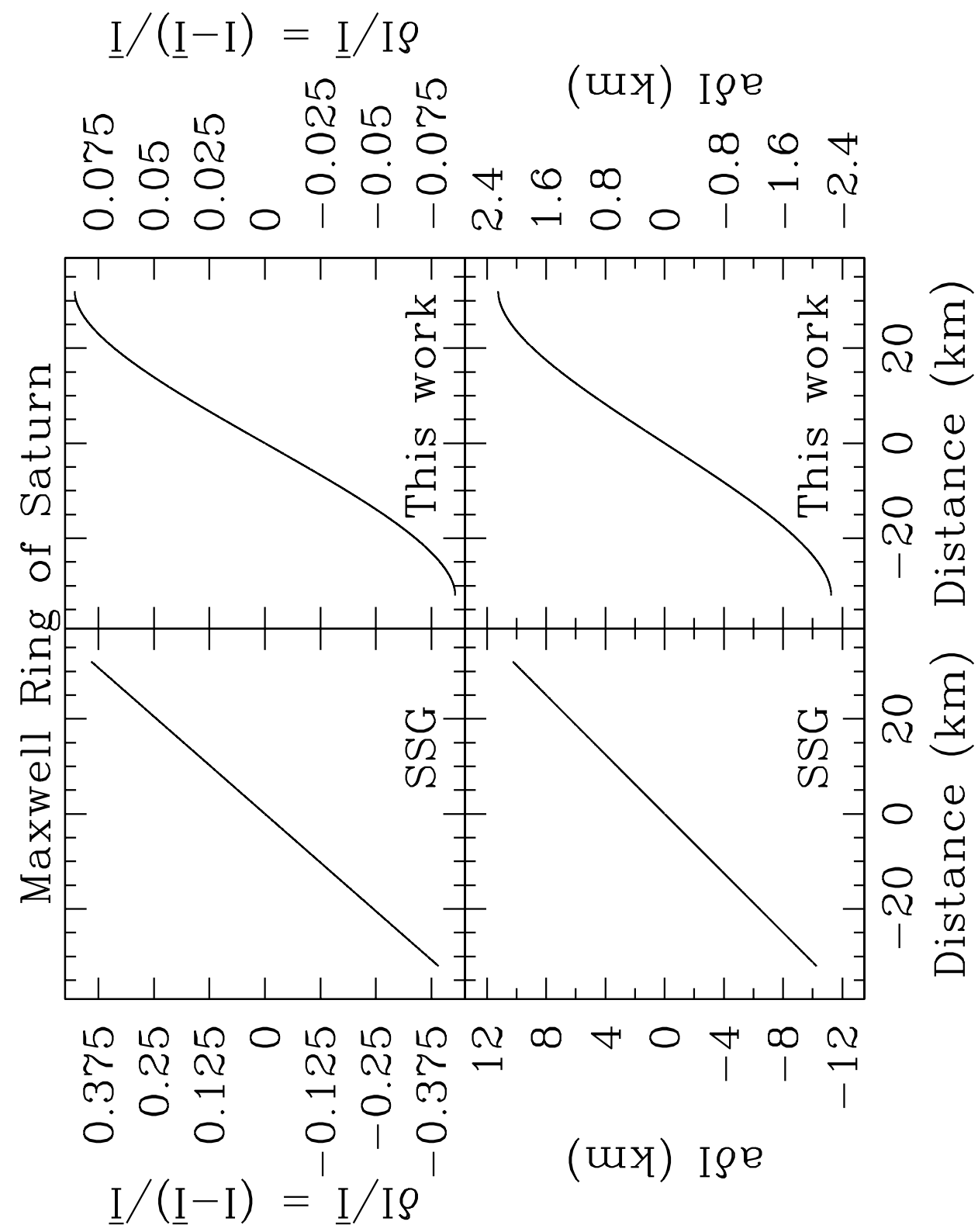

Fig. 7.- Same as Figure 5, but for the Maxwell ring of Saturn. While the mean inclination, $\bar{I}$, is unknown for the Maxwell ring, its value does not affect our calculation of the fractional variation of inclination across the ring, $(I-\bar{I}) / \bar{I}$, shown in the upper panels. By contrast, the absolute value of the vertical warp, $a \delta I=a(I-\bar{I})$, scales linearly with $\bar{I}$; to compute the values displayed in the lower panels, we have assumed $\bar{I}=3 \times 10^{-4} \mathrm{rad}$. Accounting for interparticle collisions raises the mass of the ring and therefore reduces vertical warping; the effect is less dramatic for the Maxwell ring than for the Uranian $\alpha$ ring because the radial width of the former is greater than that of the latter, so that the solution is less sensitive to boundary conditions. 


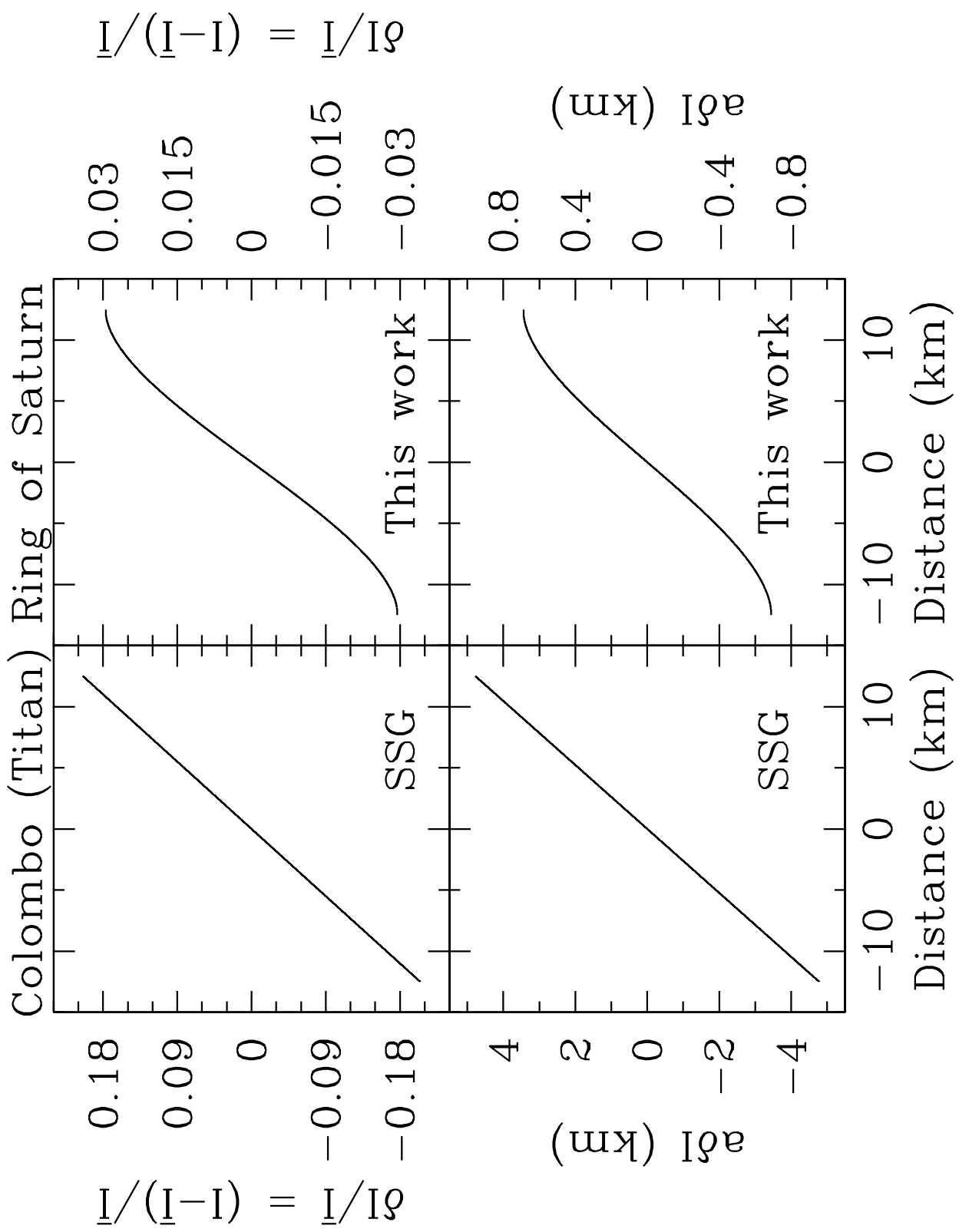

Fig. 8. - Same as Figure 7, but for the Colombo (Titan) ring of Saturn. In generating the bottom panels for $a \delta I=a(I-\bar{I})$, we have assumed $\bar{I}=3 \times 10^{-4} \mathrm{rad}$. The top panels displaying the fractional variation of inclination are independent of this assumed value. 
Note that vertical warping is substantially greater for the Saturnian Maxwell and Colombo rings than for the Uranian $\alpha$ and $\beta$ rings. Accounting for interparticle collisions near ring edges always increases ring masses and flattens ring warps, but such effects become less important as the ring width increases. The Maxwell and Titan rings are each more than $25 \mathrm{~km}$ across at quadrature, while their Uranian counterparts are less than $7 \mathrm{~km}$ across. Collisional stresses near ring edges are not as effectively communicated across wider rings.

\subsection{Neglect of Collisions in Vertical Structure}

We check a posteriori the validity of our approximation in $\S 2.2$ that interparticle collisions play no direct role in determining the inclination profile. Collisions matter most near ring edges (CG00); an upper limit for the collisional acceleration felt by a ring particle lying with a mean free path, $\lambda \sim c_{b} / \bar{n}$, of the ring edge is $c_{b}^{2} / \lambda \sim c_{b} \bar{n}$. The component of this acceleration that is perpendicular to the orbit plane of this last ring particle is $c_{b} \bar{n} q_{i}$, where $q_{I}=a(d I / d a)$ is the inclination gradient evaluated at the edge. This normal acceleration generates a rate of nodal precession (relative to the collision-induced rate on the ring midline, which is zero) of $\sim c_{b} \bar{n} q_{I} / \bar{n} \bar{a} \bar{I}=c_{b} q_{i} / \bar{a} \bar{I}$.

We divide this maximum, collision-induced differential precession rate by the differential rate of this last endwire due to the quadrupole field of the planet. For collisions to be unimportant, this dimensionless number should be less than unity. For the Uranian $\alpha$ ring, it is

$$
\mathrm{Co} \equiv \frac{8}{21} \frac{c_{b} q_{I}}{\bar{I} \bar{n} J_{2} \Delta a}\left(\frac{\bar{a}}{R_{p}}\right)^{2}=0.2\left(\frac{c_{b}}{2 \mathrm{~cm} \mathrm{~s}^{-1}}\right)\left(\frac{q_{I}}{2 \times 10^{-5}}\right),
$$

where the value for $q_{i}$ at the ring edge is obtained from our computed inclination profile. For the $\beta$ ring, we derive Co $\approx 0.2\left(\mathrm{c}_{\mathrm{b}} / 2 \mathrm{~cm} \mathrm{~s}^{-1}\right)$; for both the Saturnian Maxwell and Colombo (Titan) ringlets, $\mathrm{Co} \approx 0.1\left(\mathrm{c}_{\mathrm{b}} / 2 \mathrm{~cm} \mathrm{~s}^{-1}\right)$. Note that these values of Co for the Saturnian ringlets are independent of the assumed $\bar{I}$ since $q_{I} / \bar{I} \propto \delta I / \bar{I}$ is independent of $\bar{I}$. We conclude based on these smallish values of Co that collisions are of marginal direct importance to the inclination profiles of our ring models. As we recede from the ring edge towards the ring interior, collisions become even less important as surface density gradients decline. 


\section{STABILITY}

Here we prove that circular, nodally locked ringlets are linearly stable to perturbations to their inclinations and nodes. A corresponding proof for the stability of apsidally locked, co-planar rings would read similarly. We account not only for ring self-gravity and the planetary quadrupole field, but also for interparticle collisions, albeit in a simplistic way.

We restrict ourselves to circular rings for simplicity and for this proof only. Our hope is that accounting simultaneously for ring eccentricity would not change our conclusions. While this hope must be tempered by considerations of a time-varying $\omega$ that we have not explored in depth, we take some consolation from the Appendix in which we show how a variable $\omega$ impacts force balance in the vertical direction by seemingly modest amounts.

In $§ 3.1$, we derive the secular changes to the inclination and node of a test particle due to the gravitational influence of a nearby massive wire. In $\S 3.2$, we show how narrow rings respond to small perturbations by oscillating in a number of normal harmonic modes.

\subsection{One Wire and a Particle}

Consider the perturbations induced by a massive, circular wire on a nearby test particle. The equator plane of the planet defines the reference plane, and $\hat{\mathbf{z}}$ is the unit vector perpendicular to this plane. Let $a_{p}, I_{p}$, and $\Omega_{p}$ be the particle's semi-major axis, inclination, and longitude of ascending node, while $a, I$, and $\Omega$ refer to the corresponding orbital elements of the perturbing wire. Let $\vartheta$ denote the particle's angular position away from the node, $\vartheta=\theta-\Omega_{p}$, where $\theta$ is the particle's longitude. Define $\Delta a \equiv a-a_{p}, \Delta I \equiv I-I_{p}$, and $\Delta \Omega \equiv \Omega-\Omega_{p}$. Figure 9 illustrates the geometry.

Assume that $|\Delta a| \ll a,|\Delta I| \ll I$, and $|\Delta \Omega| \ll 1$. If the wire has total mass $m$, then its linear density is

$$
\rho=m / 2 \pi a .
$$

Because the separation between the particle and the wire is small, the wire can be approximated locally as straight. The gravitational acceleration of the particle induced by the wire is therefore

$$
\mathbf{F}=2 G \rho \mathbf{d} / d^{2},
$$

where $\mathbf{d}$ is the perpendicular vector from the particle to the wire, $d=|\mathbf{d}|$, and $G$ is the gravitational constant.

As depicted in Figure 10, the vertical distance from the particle to the wire at fixed 


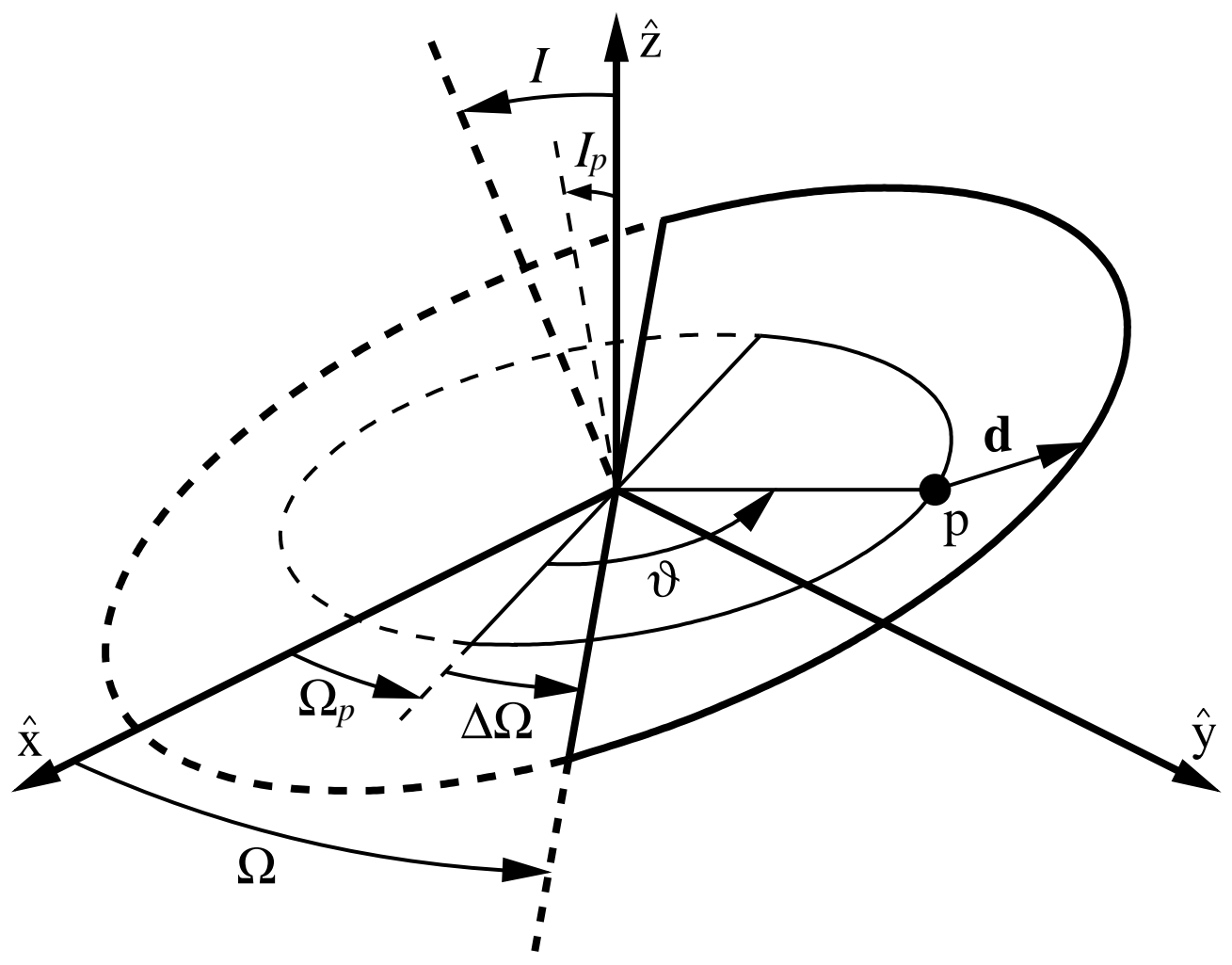

Fig. 9.- The three-dimensional geometry of the problem. The central planet rests at the origin, and the z-axis is aligned with the planet's spin axis. A test particle is located at point $\mathrm{p}$ and is displaced from its node on the planet's equator plane by an angle $\vartheta$; $\mathbf{d}$ is the perpendicular vector from the particle to the wire. Angles $\Omega$ and $I$ are the longitude of ascending node and inclination of the wire, respectively, $\Omega_{p}$ and $I_{p}$ are the corresponding angles for the particle, and $\Delta \Omega \equiv \Omega-\Omega_{p}$. The inclinations, particle-wire separation, and misalignment of nodes have all been exaggerated for clarity. 


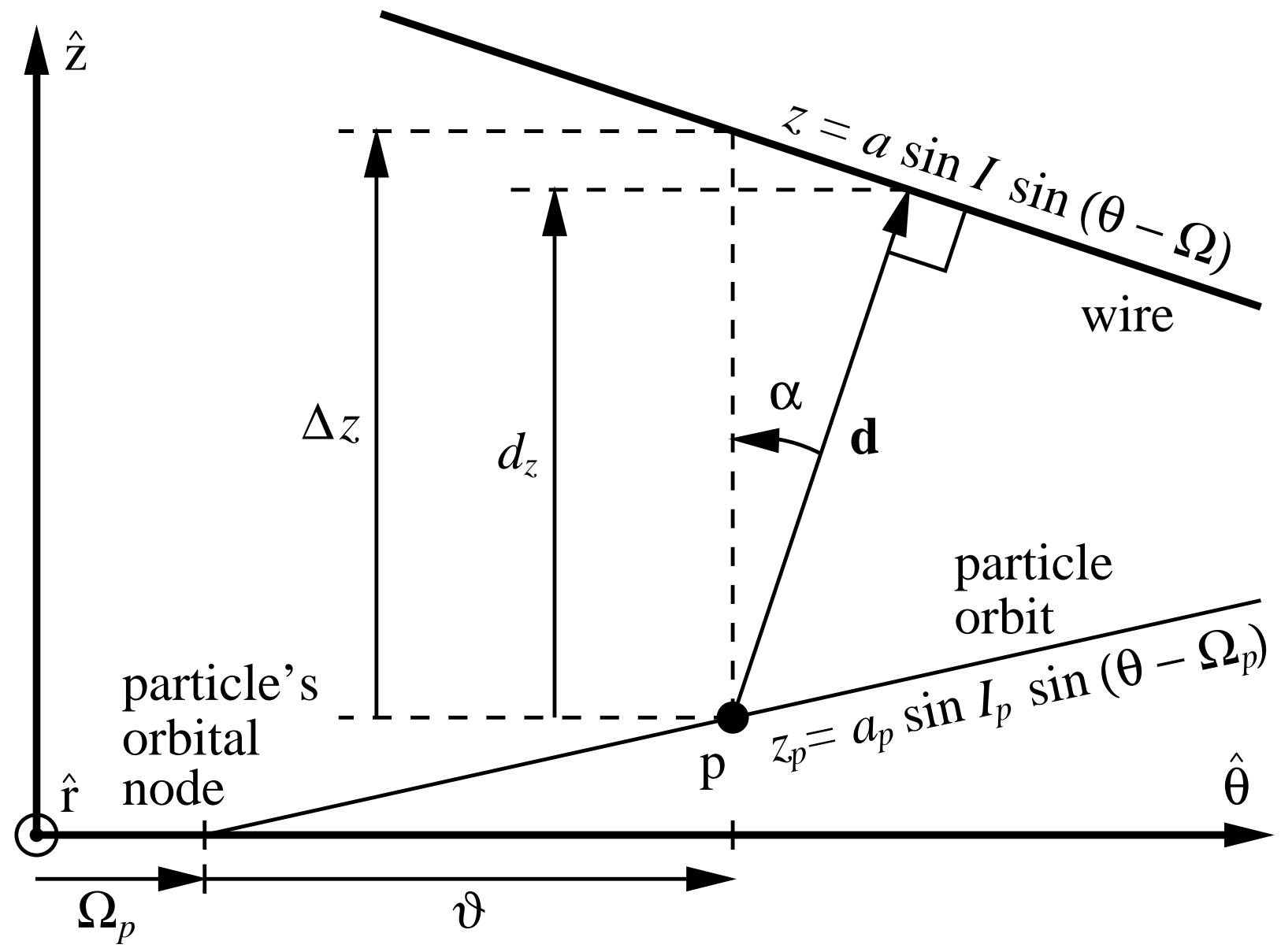

Fig. 10.- A two-dimensional projection of Figure 9 that plots height above the planet's equator plane versus longitude on that plane. To achieve an exaggerated difference in orbits, we make $\Delta \Omega$ large and negative for this figure. Because the angle $\alpha$ is $\mathcal{O}(I), d_{z}=\Delta z\left[1-\mathcal{O}\left(I^{2}\right)\right]$. 
longitude is

$$
\begin{aligned}
\Delta z & =a \sin I \sin (\vartheta-\Delta \Omega)-a_{p} \sin I_{p} \sin \vartheta \\
& =\Delta(a I) \sin \vartheta-a I \Delta \Omega \cos \vartheta
\end{aligned}
$$

where $\Delta(a I)=a I-a_{p} I_{p}$. Borderies et al. (1983b) assume $\Delta(a I) \approx a \Delta I$, which is equivalent to $\Delta a / a \ll \Delta I / I$; since we have derived inclination profiles in $\S 2.2$ for which their assumption is not valid, we keep our more accurate expression. Referring again to Figure 10, we note that since $\mathcal{O}(\alpha)=\mathcal{O}(I)$,

$$
d_{z} \equiv \mathbf{d} \cdot \hat{\mathbf{z}}=\Delta z\left[1-\mathcal{O}\left(I^{2}\right)\right]
$$

Furthermore,

$$
d^{2}=d_{z}^{2}+\Delta a^{2} .
$$

The changes in $I_{p}$ and $\Omega_{p}$ due to $\mathbf{F}$ are given to leading order by (see, e.g., Murray \& Dermott 1999)

$$
\begin{aligned}
\frac{d \Omega_{p}}{d t}(\vartheta) & =\frac{F_{z} \sin \vartheta}{n_{p} a_{p} I_{p}} \\
\frac{d I_{p}}{d t}(\vartheta) & =\frac{F_{z} \cos \vartheta}{n_{p} a_{p}},
\end{aligned}
$$

where $n_{p}$ is the particle's mean motion, $F_{z} \equiv \mathbf{F} \cdot \hat{\mathbf{z}}=2 G \rho d_{z} / d^{2} \simeq 2 G \rho \Delta z / d^{2}$ is the vertical component of the perturbing force, and we have neglected terms of order $I^{2}$. Substituting eqs. (16), (17), (18), and (20) into (21a) and (21b) yields

$$
\begin{aligned}
& \frac{d \Omega_{p}}{d t}(\vartheta)=C \frac{\frac{\Delta(a I)}{a I} \sin ^{2} \vartheta-\Delta \Omega \sin \vartheta \cos \vartheta}{1+\left(\frac{\Delta(a I)}{\Delta a} \sin \vartheta-\frac{a I \Delta \Omega}{\Delta a} \cos \vartheta\right)^{2}}, \\
& \frac{d I_{p}}{d t}(\vartheta)=C \frac{\frac{\Delta(a I)}{a} \sin \vartheta \cos \vartheta-I \Delta \Omega \cos ^{2} \vartheta}{1+\left(\frac{\Delta(a I)}{\Delta a} \sin \vartheta-\frac{a I \Delta \Omega}{\Delta a} \cos \vartheta\right)^{2}},
\end{aligned}
$$

where

$$
C=\frac{m}{\pi M_{P}}\left(\frac{a}{\Delta a}\right)^{2} n
$$

and $n$ is the mean motion at semi-major axis $a$. Where appropriate, we have neglected differences between $a_{p}$ and $a$. 
To obtain the secular changes in $I_{p}$ and $\Omega_{p}$, we average $d \Omega_{p} / d t$ and $d I_{p} / d t$ over one full

orbit of the test particle; to wit, $X=(1 / 2 \pi) \int_{0}^{2 \pi} X(\vartheta) d \vartheta$. To simplify this integral, first define $Q$ and $\gamma$ such that

$$
\begin{aligned}
Q \cos \gamma & =\frac{\Delta(a I)}{\Delta a} \\
Q \sin \gamma & =\frac{a I \Delta \Omega}{\Delta a}
\end{aligned}
$$

Then eqs. (22a) and (22b) can be re-written

$$
\begin{aligned}
& \frac{d \Omega_{p}}{d t}(\vartheta)=C\left(\frac{\Delta a}{a I}\right) \frac{Q \sin \vartheta \sin (\vartheta-\gamma)}{1+Q^{2} \sin ^{2}(\vartheta-\gamma)} \\
& \frac{d I_{p}}{d t}(\vartheta)=C\left(\frac{\Delta a}{a}\right) \frac{Q \cos \vartheta \sin (\vartheta-\gamma)}{1+Q^{2} \sin ^{2}(\vartheta-\gamma)}
\end{aligned}
$$

Averaging these equations over $\vartheta$ yields

$$
\begin{aligned}
& \left(\frac{d \Omega_{p}}{d t}\right)_{G}=C\left(\frac{\Delta a}{a I}\right) H\left(-Q^{2}\right) Q \cos \gamma=C H\left(-Q^{2}\right)\left(\frac{\Delta I}{I}+\frac{\Delta a}{a}\right) \\
& \left(\frac{d I_{p}}{d t}\right)_{G}=-C\left(\frac{\Delta a}{a}\right) H\left(-Q^{2}\right) Q \sin \gamma=-C H\left(-Q^{2}\right) I \Delta \Omega,
\end{aligned}
$$

where the subscript $G$ denotes wire gravity and

$$
H\left(-Q^{2}\right)=\frac{\sqrt{Q^{2}+1}-1}{Q^{2} \sqrt{Q^{2}+1}} .
$$

The forms of $C$ and $H$ are chosen to be consistent with eq. (6) of Borderies et al. (1983b).

\subsection{N Self-Gravitating Wires with Oblate Planet and Collisions}

Now consider the interaction between $N$ circular wires. We demonstrate that the equilibrium configuration of the wires is linearly stable. For this purpose, we do not take the wires to be equally spaced in semi-major axis, but rather define their spacing such that all wires have equal mass; the wires must therefore be more closely packed in regions of higher surface density. This de-composition by mass rather than by semi-major axis is critical to our proof of stability. Denote the total ring mass by $M$, so that each wire has mass $M / N$. Let wire $j$ have semi-major axis $a_{j}$, longitude of ascending node $\Omega_{j}$, and inclination $I_{j}$. Define mean elements by $\bar{a} \equiv \sum a_{j} / N, \bar{\Omega} \equiv \sum \Omega_{j} / N$, and $\bar{I} \equiv \sum I_{j} / N$; let $\bar{n}$ denote the Keplerian mean motion at semi-major axis $\bar{a}$. As before, we assume that variations in orbital elements 
across the ring are small: for any wire $j,\left|a_{j}-\bar{a}\right| \ll \bar{a},\left|I_{j}-\bar{I}\right| \ll \bar{I}$, and $\left|\Omega_{j}-\bar{\Omega}\right| \ll 1$. We adapt eqs. (26a) and (26b) to write down the time rates of change of $\Omega_{j}$ and $I_{j}$ due to the gravitational attraction of wire $k$ :

$$
\begin{aligned}
& \left(\frac{d \Omega_{j}}{d t}\right)_{G(k)}=C_{j k} H\left(-Q_{j k}^{2}\right)\left(\frac{I_{k}-I_{j}}{\bar{I}}+\frac{a_{k}-a_{j}}{\bar{a}}\right) \\
& \left(\frac{d I_{j}}{d t}\right)_{G(k)}=-C_{j k} H\left(-Q_{j k}^{2}\right) I\left(\Omega_{k}-\Omega_{j}\right),
\end{aligned}
$$

where

$$
\begin{gathered}
C_{j k}=C_{k j}=\frac{M}{\pi M_{P} N}\left(\frac{\bar{a}}{a_{k}-a_{j}}\right)^{2} \bar{n} \\
Q_{j k}^{2}=Q_{k j}^{2}=\left(\frac{a_{k} I_{k}-a_{j} I_{j}}{a_{k}-a_{j}}\right)^{2}+\left(\frac{\bar{a} \bar{I}\left(\Omega_{k}-\Omega_{j}\right)}{a_{k}-a_{j}}\right)^{2} .
\end{gathered}
$$

Interparticle collisions are assimilated as follows. According to equation (2), the collisional repulsion between two neighboring wires is inversely proportional to the distance between them, and the resulting acceleration is directed oppositely to that of inter-wire gravity. This prescription implies that collisional forces act effectively as anti-gravitational forces, having the same dependence on inter-wire separation as self-gravity but with an opposite sign. Thus, we can account for collisions by prepending a factor $g_{j k}$ to the right-hand sides of eqs. (28a) and (28b). The factor $g_{j k}$ represents the effects of collisions; the particular value of $g_{j k}$ depends on the local velocity dispersion; it is always $<1$, and it is negative where collisional repulsion is stronger than gravitational attraction.

Finally, consider the central planet's quadrupole field, which causes no secular change in $\left\{I_{j}\right\}$ but induces precession of the nodes $\left\{\Omega_{j}\right\}$. The effect is described to leading order by

$$
\left(\frac{d \Omega_{j}}{d t}\right)_{Q}=-\frac{3}{2} J_{2} R_{p}^{2} \sqrt{G M_{P}} a_{j}^{-7 / 2} .
$$

A linear expansion yields

$$
\left(\frac{d \Omega_{j}}{d t}\right)_{Q}=\frac{d \bar{\Omega}}{d t}+\frac{21}{4} J_{2}\left(\frac{R_{p}}{\bar{a}}\right)^{2} \bar{n} \frac{a_{j}-\bar{a}}{\bar{a}},
$$

where $d \bar{\Omega} / d t=-\frac{3}{2} J_{2} R_{p}^{2} \sqrt{G M_{P}} \bar{a}^{-7 / 2}$.

The total rates of change of $\Omega_{j}$ and $I_{j}$ due to all other wires read as

$$
\frac{d \Omega_{j}}{d t}=\sum_{k \neq j} g_{j k} C_{j k} H\left(-Q_{j k}^{2}\right)\left(\frac{I_{k}-I_{j}}{\bar{I}}+\frac{a_{k}-a_{j}}{\bar{a}}\right)
$$




$$
\begin{gathered}
+\frac{d \bar{\Omega}}{d t}+\frac{21}{4} J_{2}\left(\frac{R_{p}}{\bar{a}}\right)^{2} \bar{n} \frac{a_{j}-\bar{a}}{\bar{a}} \\
\frac{d I_{j}}{d t}=-\sum_{k \neq j} g_{j k} C_{j k} H\left(-Q_{j k}^{2}\right) I\left(\Omega_{k}-\Omega_{j}\right) .
\end{gathered}
$$

Eq. (33b) implies that $\bar{I}$ is conserved. Now denote the equilibrium inclination of wire $j$ by $I_{j}^{0}$, and define

$$
\Phi_{j}=\frac{I_{j}-I_{j}^{0}}{\bar{I}}
$$

Equations (33a) and (33b) are expressed more concisely in terms of $\Phi_{j}$ :

$$
\begin{aligned}
\frac{d \Omega_{j}}{d t} & =\sum_{k \neq j} g_{j k} C_{j k} H\left(-Q_{j k}^{2}\right)\left(\Phi_{k}-\Phi_{j}\right)+\frac{d \bar{\Omega}}{d t} \\
\frac{d \Phi_{j}}{d t} & =-\sum_{k \neq j} g_{j k} C_{j k} H\left(-Q_{j k}^{2}\right)\left(\Omega_{k}-\Omega_{j}\right) .
\end{aligned}
$$

The terms in eq. (33a) proportional to $\left(a_{k}-a_{j}\right) / \bar{a}$ and $\left(a_{j}-\bar{a}\right) / \bar{a}$ have been absorbed into $I_{j}^{0}$ (i.e., $\Phi_{j}$ ) and $I_{k}^{0}$ (i.e., $\left.\Phi_{k}\right)$.

As a final simplification, define $N \times 1$ column vectors $\vec{x}, \vec{y}$, and $\vec{u}$ with elements $x_{j}=\Omega_{j}$, $y_{j}=\Phi_{j}$, and $u_{j}=1$. Equations (35a) and (35b) can then be written in matrix form:

$$
\begin{aligned}
\frac{d}{d t} \vec{x} & =K \vec{y}+\frac{d \bar{\Omega}}{d t} \vec{u}, \\
\frac{d}{d t} \vec{y} & =-K \vec{x},
\end{aligned}
$$

where $K$ is an $N \times N$ symmetric matrix whose off-diagonal entries are given by

$$
K_{j k}=K_{k j}=g_{j k} C_{j k} H\left(-Q_{j k}^{2}\right)
$$

and whose diagonal entries equal

$$
K_{j j}=-\sum_{k \neq j} g_{j k} C_{j k} H\left(-Q_{j k}^{2}\right) .
$$

Note that $K$ is symmetric only because we have chosen our wires to have equal mass. Furthermore, we can take $H$ to be constant because the fractional variation in this quantity is of order $Q^{2}$ times the fractional variation in $Q$, and $Q \sim q_{I} \ll 1$ from our equilbrium 
model. Finally, we assume that variations in $g_{j k}$ are small. Then $K$ is a constant matrix and eqs. (36a) and (36b) are linear.

Since $K$ is symmetric, it possesses $N$ orthogonal eigenvectors $\vec{v}_{0}, \ldots, \vec{v}_{N-1}$ with real associated eigenvalues $\chi_{0}, \ldots, \chi_{N-1}$. Inspection reveals that $\vec{v}_{0}=\vec{u}$ and $\chi_{0}=0$. Thus the complete solution to eqs. (36a) and $(36 \mathrm{~b})$ is

$$
\begin{aligned}
\vec{x} & =\sum_{n=1}^{N-1} c_{n} \vec{v}_{n} \sin \left[\chi_{n}\left(t-\phi_{n}\right)\right]+\frac{d \bar{\Omega}}{d t}\left(t-t_{0}\right) \vec{u}, \\
\vec{y} & =\sum_{n=1}^{N-1} c_{n} \vec{v}_{n} \cos \left[\chi_{n}\left(t-\phi_{n}\right)\right],
\end{aligned}
$$

where the $2 N-2$ constants $c_{1}, \ldots, c_{N-1}$ and $\phi_{1}, \ldots, \phi_{N-1}$ are determined from the initial relative inclinations and longitudes of ascending nodes. The two remaining constants are $t_{0}$ and $\bar{I}$, where $t_{0}$ is set by the mean longitude of ascending node. Equations (39a) and (39b) imply that an inclined, circular ring responds to small perturbations to the inclinations and nodes of its constituent ringlets in the presence of self-gravity, planetary oblateness, and (a simplified prescription of) interparticle collisions by oscillating in a number of normal modes.

These modes resemble the vibrations of a string with free boundary conditions and variable density. A preliminary numerical study in which we modelled the ring with $g_{j k}=1$ suggests that the fundamental (lowest, non-zero) frequency is

$$
\chi_{1} \sim \frac{2 M}{\pi M_{P}}\left(\frac{\bar{a}}{\Delta a}\right)^{2} \bar{n}
$$

where $\Delta a$ is the full width of the ring. For this mode, half the ring precesses nodally in one direction relative to the ring midline, while the other half precesses in the other direction. For the Maxwell ringlet, $2 \pi / \chi_{1} \sim 8 \mathrm{yr}$. This same study indicates that $\chi_{j}=2 \chi_{1} j$ for $j>10$. We will use this numerical model in our discussion below regarding the possibility of exciting a high order mode by using force variations that arise from a time-varying $\omega$ (see also the Appendix).

\section{SUMMARY AND DISCUSSION}

Our crude and prescriptive treatment of streamline dynamics near resonantly perturbed ring boundaries prevents us from claiming great accuracy in our computed surface density and inclination profiles. Nonetheless, we believe the following conclusions to be robust: 
1. Narrow rings have total masses of order $10^{19} \mathrm{~g}$.

2. Their surface densities increase towards their edges.

3. The Uranian $\alpha$ and $\beta$ rings are vertically warped by a height of order $10 \mathrm{~m}$. The radially wider Saturnian Maxwell and Titan rings are warped by a height of order 1 $\left(\bar{I} / 3 \times 10^{-4} \mathrm{rad}\right) \mathrm{km}$. An equivalent and more model-independent statement is that the fractional variation of inclination is order $10^{-3}$ across the Uranian ringlets and of order $10^{-1}$ across their Saturnian analogues.

4. Nodally (apsidally) locked rings are linearly stable to perturbations to their inclinations (eccentricities) and nodes (apses).

Analysis of stellar occultation data by the Cassini spacecraft may test predictions 1-3 for the Maxwell and Colombo ringlets of Saturn.

For a ring to maintain apsidal and nodal alignment, its argument of pericenter must precess. This means that where the ring is "pinched" - in other words, its pericenter, the narrowest portion of the ring, where self-gravitational and collisional forces are strongestcirculates relative to the node of the ring on the equator plane of the planet. Such circulation must introduce time-variable forces into the vertical equations of motion for the ring; these time-variable forces have a frequency of $2 \dot{\tilde{\omega}}$, or about 0.08 cycles per day for the Saturn Maxwell ringlet. Might these time-variable forces drive bending waves across the ring? First note that the driving frequency is more than 2 orders of magnitude greater the fundamental bending frequency, $\chi_{1}$. Therefore any mode that is excited will have a wavelength considerably shorter than the overall width of the ring. A preliminary study suggests that the radial wavelength of the bending mode whose natural frequency matches the driving frequency is of order one kilometer for the Saturnian ringlets, a length scale possibly within reach of the Cassini camera. We defer a more thorough investigation of this possibility, including estimates of the amplitude of the mode, to future study. In the event that Cassini finds the vertical structure of a narrow ring to vary over a period of a few weeks and over kilometer lengthscales, one might look to the periodic stresses associated with a time-varying $\omega$ for the cause. Ultimately, measurements and a theoretical understanding of any modes excited might constrain the mass of the ring independently of optical depth soundings.

We have solved for the inclination gradient of a given ring by first finding the surface density profile. A key input for this procedure was the eccentricity profile of the ring, $e(a)$, which we obtained from the (spatially averaged) observations. While this procedure is physically self-consistent, it begs the question of where the eccentricity profile originated. Another way of saying this is to note that our equilibrium solutions are not the only ones 
possible in principle. For example, the steps of our procedure could be reversed: we could begin by positing an inclination gradient, solve next for the masses required to maintain nodal alignment, and conclude by deriving the eccentricity gradient required to maintain apsidal alignment. We did not adopt this procedure because the observations supplied the eccentricity gradient and not the inclination gradient. Why narrow rings self-organize themselves to exhibit dimensionless eccentricity gradients of order $q_{e}=\bar{a} d e / d a \sim 0.5$ and dimensionless inclination gradients that are significantly smaller - of order $q_{I}=\bar{a} d I / d a \sim$ $10^{-1}-10^{-3}$ for the Saturnian cases and of order $10^{-3}-10^{-5}$ for the Uranian cases - is unclear.

Many issues remain unresolved in our understanding of narrow rings. In the more than twenty years since their discovery, we still ask the following questions:

1. Are narrow rings primordial? According to classical theories of ring shepherding, attendant satellites extend the viscous spreading time of a ring by a factor of order the mass ratio between the shepherd and the ring. This extension factor is modest for the Uranian $\epsilon$ ring, whose mass is thought to be comparable to that of its shepherds (CG00). The situation is likely to be similar for other rings, especially given the large ring masses that we are deriving. Estimates for the spreading time of the $\epsilon$ ring range from $3 \times 10^{4}$ yr to $2 \times 10^{8} \mathrm{yr}$, all of which are shorter than the age of the solar system (Chiang 2003). But if narrow rings are not formed from primordial circumplanetary disks, how can we explain the tiny mean inclinations exhibited by the Uranian rings with respect to the equator plane of their severely oblique host planet? New estimates of ring lifetimes based on non-classical theories of shepherding (see, e.g., Goldreich \& Porco 1987, and Borderies, Goldreich, \& Tremaine 1984) would be welcome.

2. Why do all narrow rings exhibit positive and not negative eccentricity gradients?

3. Why is the inclination gradient smaller in magnitude than the eccentricity gradient?

We might hope to answer these questions by modelling more carefully the distortion of streamlines near ring edges by shepherd satellites.

It is a pleasure to thank Joanne Cohn for introducing C.J.C. to E.I.C. We thank also Carolyn Porco for faxing pages from her thesis to us, and an anonymous referee for a helpful report that prompted us to examine our statements regarding bending modes more carefully. This work was supported in part by a Space Grant Summer Fellowship awarded to C.J.C. by the Space Sciences Laboratory at Berkeley. E.I.C. acknowledges support by National Science Foundation Planetary Astronomy Grant AST-0205892 and Hubble Space Telescope Theory Grant HST-AR-09514.01-A. 


\section{A. Time-varying $\omega$}

Here we explore the effect of a time-varying argument of pericenter, $\omega$, on our equation of motion (6) for nodal precession in the presence of ring self-gravity. Consider two elliptical wires orbiting a spherical planet, one placed in an arbitrary reference plane, and a second placed at an inclination, $\Delta I$, relative to that plane. Take the longitudes of pericenter of the two wires to be equal, $\tilde{\omega}_{1}=\tilde{\omega}_{2}=0$. The longitude of ascending node of the second wire is $\Omega$. How sensitive is the orbit-averaged rate of nodal precession of the second wire, $\langle\dot{\Omega}\rangle$, to $\Omega=-\omega ?$

At every true anomaly, $f$, of the second wire, the perpendicular distance between the second wire and the first is

$$
d \approx \sqrt{(\Delta a)^{2}\left(1-q_{e} \cos f\right)^{2}+a^{2}(\Delta I)^{2} \sin ^{2}(f-\Omega)}
$$

where we have assumed that the difference in semi-major axes of the wires, $\Delta a$, is much smaller than the semi-major axis of either wire, $a$. The eccentricity gradient between the wires is $q_{e} \equiv a \Delta e / \Delta a$, where $\Delta e$ is the difference in wire eccentricities. The first term under the square root in equation (A1) represents the in-plane separation between the wires, while the second term represents the out-of-plane contribution. Re-write (A1) as

$$
d \approx \Delta a \sqrt{\left(1-q_{e} \cos f\right)^{2}+q_{I}^{2} \sin ^{2}(f-\Omega)}
$$

where $q_{I} \equiv a \Delta I / \Delta a$.

By Gauss's equation, the instantaneous rate of nodal precession of a test particle whose orbit coincides with the second wire reads

$$
\dot{\Omega}=\frac{F_{z} \sin (f-\Omega)}{n a \Delta I}
$$

where $n$ equals the mean motion of the second wire, $F_{z}$ equals the vertical acceleration felt by the test particle,

$$
F_{z}=-\frac{2 G \rho a \Delta I \sin (f-\Omega)}{d^{2}}
$$

and $\rho$ equals the linear mass density of the first wire of mass $m$, 


$$
\rho=\frac{m}{2 \pi a}
$$

We have dropped corrections to (A5) due to wire eccentricity because the acceleration (A4) is already small in $\Delta I$. Time-averaging $\dot{\Omega}$ over one orbital period yields

$$
\begin{gathered}
\langle\dot{\Omega}\rangle=-\frac{m}{\pi M_{P}} n \frac{a}{\Delta a} \frac{q_{I}}{\Delta I} B\left(q_{e}, q_{I}, \Omega\right), \\
B=\frac{1}{2 \pi} \int_{0}^{2 \pi} \frac{\sin ^{2}(f-\Omega)}{\left(1-q_{e} \cos f\right)^{2}+q_{I}^{2} \sin ^{2}(f-\Omega)} d f .
\end{gathered}
$$

Equation (A6) is identical in form to equation (6). The sensitivity of $\langle\dot{\Omega}\rangle$ to $\omega=-\Omega$ is contained in the integral, $B$. Numerical integration of (A7) reveals that $B \in[0.613,0.896]$ for $q_{e}=0.5$ and $q_{I}=0.1$. If $q_{e}=0.5$ and $q_{I}=0$, then $B \in[0.619,0.920]$. The variation of force with varying $\omega$ is restricted to less than a factor of 1.5 . In $\S 2$, we fixed $B=0.77$ for a maximum $24 \%$ fractional error; the error so accrued is less than the error introduced by our prescriptions for force balance near ring boundaries. However, the force variation is large enough that it may excite observable short-wavelength bending waves across the ring, as we discuss in $\S 4$. 


\section{REFERENCES}

Borderies, N., Goldreich, P., \& Tremaine 1982, Nature, 299, 209

Borderies, N., Goldreich, P., \& Tremaine, S. 1983a, AJ, 88, 226

Borderies, N., Goldreich, P., \& Tremaine, S. 1983b, AJ, 88, 1560

Borderies, N., Goldreich, P., \& Tremaine, S. 1984, in Planetary Rings, eds. R. Greenberg \& A. Brahic (Tucson: University of Arizona Press), 713

Chiang, E.I. 2003, ApJ, 584, 465

Chiang, E.I., \& Goldreich, P. 2000, ApJ, 540, 1090 (CG00)

Elliot, J.L., \& Nicholson, P.D. 1984, in Planetary Rings, eds. R. Greenberg \& A. Brahic (Tucson: University of Arizona Press), 25

French, R.G., Nicholson, P.D., Porco, C.C., \& Marouf, E.A. 1991, in Uranus, eds. J.T. Bergstrahl, E.D. Miner, \& M.S. Matthews (Tucson: University of Arizona Press), 327

Goldreich, P., \& Tremaine, S. 1979a, Nature, 277, 97

Goldreich, P., \& Tremaine, S. 1979b, AJ, 84, 1638

Goldreich, P., \& Porco, C.C. 1987, AJ, 93, 730

Mosqueira, I., \& Estrada, P.R. 2002, Icarus, 158, 545

Murray, C.D, \& Dermott, S.F. 1999, Solar System Dynamics (New York: Cambridge University Press)

Porco, C. C. 1983, Ph.D. thesis

Porco, C. C. 1990, Adv. Space Res., 10, 221

Press, W. H., Teukolsky, S. A., Vetterling, W. T., \& Flannery, B. P. 1992, Numerical Recipes in Fortran (Cambridge : Cambridge University Press) 
Table 1. Observed and Derived Parameters of Narrow Eccentric Rings

\begin{tabular}{cccccccccc}
\hline \hline Ring & Planet & $\bar{a}(\mathrm{~km})^{\mathrm{a}}$ & $\Delta a(\mathrm{~km})^{\mathrm{a}}$ & $\bar{e}\left(\times 10^{3}\right)^{\mathrm{a}}$ & $\left(\bar{q}_{e}\right)^{\mathrm{a}}$ & $\bar{I}\left(\times 10^{3}\right)^{\mathrm{a}}$ & $\left(\bar{q}_{I}\right)^{\mathrm{b}}$ & $(\Delta I / \bar{I})^{\mathrm{b}}$ & $M\left(10^{19} \mathrm{~g}\right)^{\mathrm{b}}$ \\
\hline$\alpha$ & Uranus & 44718 & 7.15 & 0.761 & 0.472 & 0.265 & 0.00095 & 0.00057 & 0.467 \\
$\beta$ & Uranus & 45661 & 8.15 & 0.442 & 0.370 & 0.089 & 0.00037 & 0.00075 & 0.495 \\
Maxwell & Saturn & 87491 & 64 & 0.34 & 0.46 & $(0.3)$ & $(0.070)$ & 0.17 & 5.00 \\
Colombo (Titan) & Saturn & 77871 & 25 & 0.26 & 0.44 & $(0.3)$ & $(0.055)$ & 0.059 & 1.64 \\
& & & & & & & & & \\
\hline
\end{tabular}

${ }^{a}$ Uranian ring values taken from Tables I and VII of French et al. (1991). Saturnian ring values taken from Table IIId of Porco (1983) and Table 1 of Porco (1990). Values for $\bar{I}$ in parentheses are guessed.

${ }^{\mathrm{b}}$ Derived from this work. Values in parentheses for $\bar{q}_{I} \equiv \bar{a} \Delta I / \Delta a$, where $\Delta I$ is the difference in orbital inclinations between inner and outer ring edges, scale linearly with the guessed value of $\bar{I}$.

Table 2. Theoretical Model Parameters

\begin{tabular}{cccccc}
\hline \hline Ring & $c_{i}\left(\mathrm{~cm} \mathrm{~s}^{-1}\right)$ & $c_{b}\left(\mathrm{~cm} \mathrm{~s}^{-1}\right)$ & $w_{r}(\mathrm{~km})^{\mathrm{a}}$ & $\lambda(\mathrm{km})^{\mathrm{b}}$ & $N$ \\
\hline$\alpha$ & 0.1 & 2.0 & 0.44 & 0.079 & 1000 \\
$\beta$ & 0.1 & 2.0 & 0.45 & 0.081 & 1000 \\
Maxwell & 0.1 & 3.0 & 0.56 & 0.126 & 3000 \\
Colombo (Titan) & 0.1 & 2.0 & 0.50 & 0.071 & 3000 \\
& & & & & \\
\hline
\end{tabular}

a Resonant width, computed as $w_{r}=\bar{a} \sqrt{M_{\text {sat }} / M_{P}}$, where the mass of an individual (as yet unobserved) shepherd satellite equals $M_{\text {sat }}=8.4 \times 10^{18} \mathrm{~g}$ for the Uranian ringlets and $M_{\text {sat }}=2.3 \times 10^{19} \mathrm{~g}$ for the Saturnian ringlets.

${ }^{\mathrm{b}}$ Mean free path near ring edge, computed as $\lambda=c_{b} / \bar{n}$, where $\bar{n}$ is the average mean motion of the ring. 